\section{Mirrors to halt global warming?}

SIR-It seems very likely that little will be done in the future to reduce the emission of greenhouse gases. If global warming is confirmed, a remote but feasible possibility to reverse the temperature increase is by changing artificially the solar radiation incident on the Earth.

The radiation balance of the Earth is controlled by the Stefan-Boltzmann law:

$$
\varepsilon \sigma T^{4}=(1-A) S_{0} / 4
$$

where the left-hand side is the infrared black-body radiation from the Earth and the right-hand side represents the mean solar (visible) radiation on the Earth. Here $A(=0.3)$ is the albedo of the Earth, $S_{0}\left(=1.372 \mathrm{~kW} \mathrm{~m}^{-2}\right)$ is the solar constant near the Earth, $\sigma\left(=5.67 \times 10^{-8} \mathrm{~W} \mathrm{~m}^{-2} \mathrm{~K}^{4}\right)$ is the Stefan-Boltzmann constant, and $\varepsilon(=0.62)$ is the emissivity of the Earth with its atmosphere. Equation (1) gives $T=288 \mathrm{~K}$ as the mean temperature on the

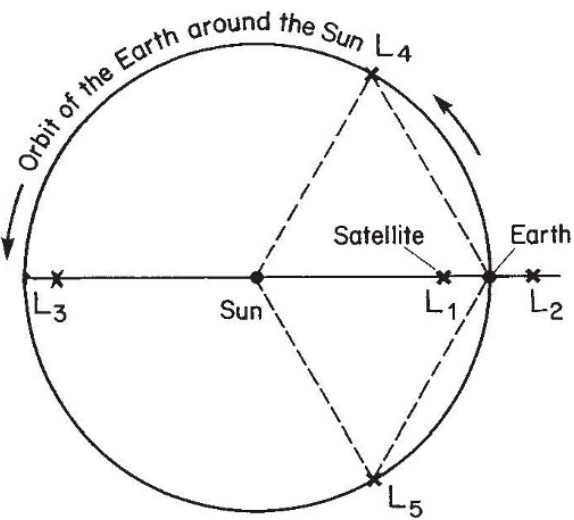

The five Lagrange points $L_{1}-L_{5}$ for the threebody system (Sun, Earth and satellite). In our case $L_{1}$ is chosen as the position for the mirrorbearing satellite.

Earth's surface.

To compensate for a temperature increase of $\Delta T$ originating from the greenhouse effect, we have to reduce the relative radiation $\Delta S_{0}$ by

$$
\frac{\Delta S_{0}}{S_{0}}=4 \frac{\Delta T}{T}
$$

For example, to compensate for a temperature increase of $2.5 \mathrm{~K}$, the solar radiation must be reduced by about $3.5 \%$. The task could be done by satellites bearing large lightweight mirrors. However, satellites in 'normal' orbits do not cast their shadows permanently on the Earth.

This difficulty may be overcome, and the $3.5 \%$ reduction achieved, with a minimum mirror area of $4.5 \times 10^{6} \mathrm{~km}^{2}$, by positioning a satellite in such a way that it will always stand between the Sun and the Earth, permanently casting its shadow on the Earth. In this context my attention has been drawn to some particular solutions of the three-body problem, the so-called Lagrange points.
In the plane of the elliptical orbit of a planet around the Sun there are five Lagrange points, $\mathrm{L}_{1}$ to $\mathrm{L}_{5}$ (see figure), which are characterized by the fact that if a third body (a satellite, for example) is positioned at one of these points, the geometrical figure between the body, the Sun and the Earth remains constantly selfsimilar. For our purpose the so-called collinear solutions, based on $\mathrm{L}_{1}, \mathrm{~L}_{2}$ and $\mathrm{L}_{3}$, are the most important, particularly Lagrange point $\mathrm{L}_{1}$. If a satellite was positioned at $\mathrm{L}_{1}$ it would remain on a straight line between the Sun and the Earth at all times and could cast its shadow permanently on the Earth. Thus, this would make possible a reduction of the solar radiation power on the Earth using a minimum of mirrors.

If we assume that the mass of the satellite is negligible relative to the mass of the Earth, then the distance between $\mathrm{L}_{1}$ and the Earth is $d \approx 1.5 \times 10^{6} \mathrm{~km}$, or about four times the distance between the Moon and the Earth (K. Stumpff Himmelsmechanik Vol. II, 104; VEB, Berlin, 1965). The satellite would have an infinite synodical period and its sidereal period would be the same as the Earth's (that is, one year).

How much energy will be required to bring mirrors of the necessary size to the point $L_{1}$ ? If we assume that they consist of aluminium of mass $10 \mathrm{~g} \mathrm{~m}^{-2}$, at least 45 million tonnes of material will have to be

\section{Drying, $\mathrm{O}_{2}$ and mass extinction}

SIR-Reports ${ }^{1,2}$ of a drop in the ${ }^{13} \mathrm{C} /{ }^{12} \mathrm{C}$ ratio of the oceans during the late Permian have been interpreted as a result of an input of light carbon to the oceans from the oxidative weathering of pre-existing organic matter, either on the ocean floor ${ }^{2}$ or on the continents ${ }^{1}$. Increased weathering on the continents is attributed to the lowering of sea level and the exposure of additional organic-containing sediments to the atmosphere at that time'. Weathering of organic matter results in the uptake of $\mathrm{O}_{2}$ from the atmosphere, and a lowering of atmospheric oxygen might have contributed to the Permo/Triassic extinction ${ }^{2}$, the most extensive mass extinction to occur over all of Phanaerozoic time ${ }^{3}$.

Although increased organic oxidation during the latest Permian might have been important, another factor which should have contributed to a more gradual lowering of atmospheric oxygen during the midto-late Permian, is the general trend towards greater continental aridity, resulting in a lowering of worldwide organic matter burial. Burial of organic matter in sediments represents net photosynthesis and net oxygen production; thus, any brought to $\mathrm{L}_{1}$. We estimate that the energy required to do this is equivalent to the output of 30 nuclear power stations producing $1 \mathrm{GW}$ for 20 years. A very crude estimate of the cost of this venture gives, over 20 years, a figure of $6 \%$ of the present annual gross national product of the world per year, which is roughly equivalent to its total annual military expenditure.

It should be noted that the Lagrange position $\mathrm{L}_{1}$ is an unstable position: there is no negative feedback mechanism to hold the satellite in position should it drift away for any reason, for example because of the light pressure of the Sun on the mirror. Furthermore, the Lagrange solutions are strictly valid only for a pure three-body problem. Although the attractive force of the moon on $\mathrm{L}_{1}$ is at most only $2 \%$ of that of the Earth, this will have some influence. Thus the satellite would have to be stabilized actively, which requires permanent observation and maintenance. However, the solar light pressure is just strong enough to allow a robust active control of the equilibrium of the mirror.

Of course, there are other important aspects which have not been considered, the most critical of which is whether it is possible to produce reflecting foils with a mass of only $10 \mathrm{~g} \mathrm{~m}^{-2}$ which will be able to withstand mechanical forces, solar winds and so on for a long time.

\section{Chapfstrasse 4}

WALTER SEIFRITZ

\section{$\mathrm{CH}-5200$ Windisch, Switzerland}

large drop in worldwide organic burial should result in a drop in atmospheric $\mathrm{O}_{2}$.

Because of the rise of vascular land plants and the deposition of their remains in terrestrial coal swamps and, by means of river transport, in the oceans, there was a considerable increase of worldwide organic matter deposition during the Carboniferous and Permian periods compared with earlier times. This conclusion is based on independent modelling of the carbon and sulphur isotope composition of marine sediments ${ }^{4-6}$ and of the relative abundance of different types of sedimentary rocks ${ }^{7}$. (Isotope changes resulting from burial of organic carbon on land are sensed by the oceans by means of carbon transport to the sea by rivers and the atmosphere.)

One can combine organic burial rates with various assumptions about organic weathering to calculate changes in the level of atmospheric oxygen. The figure shows an example for the late Palaeozoic/ early Mesozoic, based on the rockabundance model ${ }^{7}$. Note the high sensitivity of atmospheric $\mathrm{O}_{2}$ concentration to the value assumed for the average total organic carbon concentration in coalbasin sediments. A reasonable average value for the concentration of total organic carbon, present both as coal plus 